From the Departments of Surgery, Medicine, and Epidemiology and Biostatistics, Memorial Sloan-Kettering Cancer Center, New York, NY

Submitted November 12, 2008; accepted January 22, 2009; published online ahead of print at www.jco.org on June 1, 2009

Authors' disclosures of potential conflicts of interest and author contributions are found at the end of this article.

Corresponding author: Philip B. Paty, MD, Colorectal Service, Department of Surgery, Memorial Sloan-Kettering Cancer Center, 1275 York Ave, New York, NY 10065; e-mail: patyp@ mskcc.org.

The Acknowledgment is included in the full-text version of this article, available online at www.jco.org. It is not included in the PDF version (via Adobe $®$ Reader $®$ )

(C) 2009 by American Society of Clinical Oncology

0732-183X/09/2720-3379/\$20.00

DOI: $10.1200 / J C O .2008 .20 .9817$

\title{
Outcome of Primary Tumor in Patients With Synchronous Stage IV Colorectal Cancer Receiving Combination Chemotherapy Without Surgery As Initial Treatment
}

George A. Poultsides, Elliot L. Servais, Leonard B. Saltz, Sujata Patil, Nancy E. Kemeny, Jose G. Guillem, Martin Weiser, Larissa K.F. Temple, W. Douglas Wong, and Phillip B. Paty

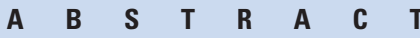

\section{Purpose}

The purpose of this study was to describe the frequency of interventions necessary to palliate the intact primary tumor in patients who present with synchronous, stage IV colorectal cancer (CRC) and who receive up-front modern combination chemotherapy without prophylactic surgery.

\section{Patients and Methods}

By using a prospective institutional database, we identified 233 consecutive patients from 2000 through 2006 with synchronous metastatic CRC and an unresected primary tumor who received oxaliplatin- or irinotecan-based, triple-drug chemotherapy (infusional fluorouracil, leucovorin, and oxaliplatin; bolus fluorouracil, leucovorin, and irinotecan; or fluorouracil, leucovorin, and irinotecan) with or without bevacizumab as their initial treatment. The incidence of subsequent use of surgery, radiotherapy, and/or endoluminal stenting to manage primary tumor complications was recorded.

\section{Results}

Of 233 patients, 217 (93\%) never required surgical palliation of their primary tumor. Sixteen patients $(7 \%)$ required emergent surgery for primary tumor obstruction or perforation, 10 patients $(4 \%)$ required nonoperative intervention (ie, stent or radiotherapy), and $213(89 \%)$ never required any direct symptomatic management for their intact primary tumor. Of those 213 patients, 47 patients $(20 \%)$ ultimately underwent elective colon resection at the time of metastasectomy, and eight patients $(3 \%)$ underwent this resection during laparotomy for hepatic artery infusion pump placement. Use of bevacizumab, location of the primary tumor in the rectum, and metastatic disease burden were not associated with increased intervention rate.

\section{Conclusion}

Most patients with synchronous, stage IV CRC who receive up-front modern combination chemotherapy never require palliative surgery for their intact primary tumor. These data support the use of chemotherapy, without routine prophylactic resection, as the appropriate standard practice for patients with neither obstructed nor hemorrhaging primary colorectal tumors in the setting of metastatic disease.

\section{J Clin Oncol 27:3379-3384. (C) 2009 by American Society of Clinical Oncology}

\section{INTRODUCTION}

In the absence of symptoms (ie, bleeding, perforation, obstruction) or resectable metastatic disease, primary tumor resection in patients who present with metastatic colorectal cancer is of uncertain benefit. The rationale for immediate resection in asymptomatic patients is based on the prevention of primary-related complications later during the treatment course, which can lead to urgent surgery associated with higher mortality. Advocates of the deferred surgical approach argue that surgery at diagnosis can delay or even preclude systemic chemotherapy and that most patients will never develop symptoms; therefore, patients should be spared unnecessary operations.

With recent advances in systemic chemotherapy and improvement in the survival of patients with stage IV colorectal cancer, the risks and benefits of a deferred surgical strategy have not been completely evaluated. In fact, combinations of infusional fluorouracil/leucovorin with oxaliplatin or irinotecan have yielded response rates of $50 \%$, disease control rates of $85 \%$, and a median survival rate of 20 months in prospective clinical trials. ${ }^{1,2}$ Furthermore, the addition of the antiangiogenic agent bevacizumab to the above combinations has provided clinically meaningful improvement in response 
rates and survival for these patents. ${ }^{3-5}$ As the assumption that colorectal cancer is a chemorefractory disease is no longer valid, the efficacy of modern chemotherapy in provision of local control of the primary tumor in the setting of metastatic disease remains to be determined but can be expected to be quite high.

The objective of our study was to describe the incidence of primary tumor-related complications, which required operative or nonoperative intervention, in patients who presented with synchronous, stage IV colorectal cancer and who received up-front modern, tripledrug, oxaliplatin- or irinotecan-based combination chemotherapy with or without bevacizumab.

\section{PATIENTS AND METHODS}

\section{Cohort Definition}

We used the Memorial Sloan-Kettering Cancer Center (MSKCC) Institutional Database (IDB) to identify all patients who met the following criteria: presentation to MSKCC with synchronous, stage IV colorectal cancer between January 1, 2000 and December 31, 2006; no previous primary tumor-directed surgery, stenting, or radiotherapy; up-front, first-line chemotherapy that consisted of bolus fluorouracil, leucovorin, and irinotecan, ${ }^{6}$ infusional fluorouracil, leucovorin, and oxaliplatin (FOLFOX), ${ }^{7,8}$ or infusional fluorouracil, leucovorin, and irinotecan ${ }^{9}$; and observation at our institution.

\section{Data Sources}

Patients were identified through the MSKCC IDB, a central data repository that is expanded prospectively on a daily basis with clinical, financial, operational, and research data. Data available within the IDB include patient demographics, histologic diagnoses that are based on International Classification of Diseases coding standards, initial American Joint Committee on Cancer stages, physician and hospital billing data, inpatient admission and outpatient registration data, operating room procedures, laboratory results, and computerized pharmacy records. The IDB undergoes continuous crossplatform integration with the MSKCC Cancer Registry. ${ }^{10}$

Additionally, inpatient and outpatient records of all identified patients were reviewed individually to confirm the presence of an intact primary tumor (that had not been previously resected, diverted, radiated, endoscopically stented, or ablated), the histologic diagnosis of colorectal adenocarcinoma, the type and duration of chemotherapy administered, sites of metastatic disease at presentation, laboratory data at presentation (ie, albumin, carcinoembryonic antigen, lactate dehydrogenase, and alkaline phosphatase), primary tumorrelated complications that required surgery (ie, resection or diversion) or nonsurgical interventions (ie, endoluminal stenting, radiotherapy, endoscopic ablative techniques), incidence of resection for preemptive palliation (ie, before the onset of symptoms) or with curative intent, and vital status at last follow-up. The resectability status of metastatic disease at presentation was not recorded, as the operative criteria for metastasectomy for colorectal cancer evolved substantially during the study period. ${ }^{11}$

All patients were asymptomatic at initial consultation with regard to their primary tumors (ie, no major bleeding, perforation, impending obstruction). Anemia, weight loss, or right upper quadrant abdominal pain (secondary to expansion of Glisson's capsule from underlying liver metastases) were not considered direct manifestations of the primary tumor. A waiver of authorization to conduct this study was obtained by the MSKCC institutional review board.

\section{Statistical Analysis}

Demographic and clinical characteristics were summarized by medians for continuous variables and by counts and percentages for categoric variables. Estimates of median survival were generated by using the Kaplan-Meier method. One of the study objectives was to investigate whether the need for emergent intervention adversely affected survival. In this data set, 26 patients had emergent interventions. Because these were performed at various times after initiation of chemotherapy, the presence of an emergent intervention was treated as a time-dependent covariate in a Cox model. For each patient who had such an intervention, the median time from chemotherapy to intervention and the Kaplan-Meier estimates for median time from intervention to death also were calculated. Estimates are reported with $95 \%$ CIs. To examine what clinical and demographic characteristics were associated with the need for emergent intervention, patients who had documented emergent interventions were compared with all other patients by using the Fisher's exact test for binary variables and the Wilcoxon rank sum test for continuous variables.

\section{RESULTS}

\section{Patient Characteristics}

Two hundred thirty-three consecutive patients were identified on the basis of the study criteria. The median age was 60 years (range, 26 to 86 years). Primary tumors were evenly distributed between the right colon (cecum, ascending, or transverse colon) in 87 patients (37\%), the left colon (descending or sigmoid) in 68 patients (29\%), and the rectum in 78 patients $(34 \%)$. The most common site of metastatic disease at presentation was the liver (221 patients [95\%]), followed by retroperitoneal nodes (91 patients [39\%]), lung (70 patients [30\%]), peritoneum (22 patients [9\%]), skeleton (four patients $[2 \%]$ ), and brain (one patient [0.4\%]). Metastatic disease involved only one site or organ in 94 patients (40\%), two sites in 106 patients $(45 \%)$, three sites in 29 patients $(12 \%)$, and four sites in four patients $(2 \%)$.

\section{Chemotherapy}

The first-line regimen was oxaliplatin based (ie, FOLFOX) in 139 patients $(60 \%)$ and irinotecan based (ie, bolus fluorouracil, leucovorin, and irinotecan or infusional fluorouracil, leucovorin, and irinotecan) in 94 patients (40\%). The median duration of the first-line regimen was 22 weeks (range, 0 to 127 weeks). Two patients received only one dose of chemotherapy, and deterioration of their performance status precluded additional treatment. Bevacizumab was used as part of the first-line regimen in 112 patients (48\%). One hundred sixty-two patients (70\%) received two or more lines of chemotherapy.

\section{Primary Tumor-Directed Interventions}

Figure 1 illustrates the outcomes of unresected primary tumors in the 233 patients studied. Of the entire cohort, 217 patients (93\%) never required surgery for primary tumor-related symptoms. Sixteen (7\%) underwent emergent surgery, including eight resections and eight diversion procedures. Five of the eight resections were performed for perforation, all at the site of the primary tumor. The remaining three resections, as well as the eight diversion procedures (seven ostomy creations and one ileocolic bypass), were performed for primary tumor obstruction. No patient in this series required any other cancer-related abdominal operation, such as for the management of small bowel obstruction as a result of peritoneal or retroperitoneal disease progression. Median time from initiation of chemotherapy to surgical intervention was 7 months (range, 1 to 27 months; Table 1). There were no episodes of proximal colonic perforation secondary to more distally obstructing carcinoma or of intractable bleeding that necessitated surgical intervention. Of five tumor perforations, two occurred while the patients were on bevacizumab treatment ( 2 and 8 months into therapy, respectively), one occurred 6 


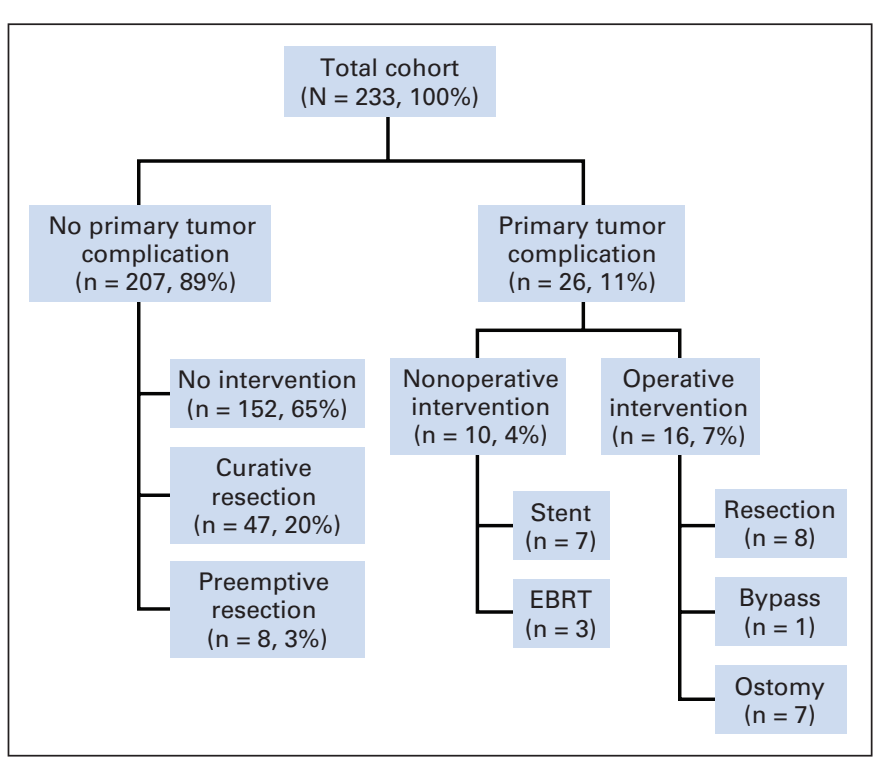

Fig 1. Outcomes of unresected primary tumor in 233 patients with synchronous stage IV colorectal cancer who received modern combination chemotherapy at Memorial Sloan-Kettering Cancer Center from 2000 to 2006. Curative resections were elective, combined resections of colorectal tumor and metastatic disease. Preemptive resections were performed in asymptomatic patients undergoing hepatic artery infusion pump placement. EBRT, external-beam radiation therapy for rectal cancer palliation.

months after the last dose of bevacizumab, and two occurred in patients who had never received bevacizumab (at 1 and 10 months after initiation of chemotherapy, respectively).

Ten patients in the study (4\%) developed primary tumor-related symptoms that were managed nonoperatively at a median time of 12 months (range, 1 to 36 months; Table 1). Endoluminal stenting was successful in seven patients, but repeat stent insertion was required in three because of tumor ingrowth or stent migration. In addition, three of seven patients who eventually underwent diverting ostomy creation had a previous failed stent insertion. Laser recanalization of nearobstructing rectal tumors was utilized to facilitate subsequent endoluminal stenting in two patients. External-beam radiation therapy was used to palliate perineal pain in three patients with metastatic rectal cancer.

Of the 217 (93\%) patients who never required emergent surgery, 47 (20\% of entire cohort) eventually underwent elective curative resection of their primary tumors and metastatic disease at a median time of 8 months (range, 5 to 32 months; Table 1) from initiation of chemotherapy. Eight (3\% of entire cohort) underwent preemptive resection before the onset of symptoms concurrent with hepatic artery infusion pump placement for regional liver chemotherapy at a median time of 9 months (range, 5 to 24 months; Table 1). Interestingly, seven of the 47 curatively resected tumors and one of the eight preemptively resected tumors demonstrated complete response to chemotherapy on pathologic examination.

\section{Surgical Mortality and Overall Survival}

Median overall survival for the entire patient cohort was 18 months from initiation of systemic chemotherapy (95\% CI, 16 to 20 months). No patient who underwent curative or preemptive elective resection died within 30 days of surgery. However, of the 16 patients who underwent emergent surgery, two $(12.5 \%)$ died in the 30 -day postoperative period, which represented $0.8 \%$ of the total study population. When these two patients were included, median survival after surgical intervention was 6 months, whereas median survival after nonsurgical intervention was 7 months (Table 1). Median overall survival for the 152 patients who never required intervention was 13 months from the initiation of chemotherapy, which is comparable with survival from initiation of chemotherapy for the 26 patients who eventually needed intervention (by addition of time to procedure and survival after procedure; Table 1). In addition, when included as a time-varying covariate in a Cox regression model, the need for emergent intervention did not correlate with overall sur$\operatorname{vival}(P=.81)$.

We sought to identify clinical or laboratory variables at presentation that could be associated with an increased intervention rate. The vast majority of patients in this analysis (211 of 233, or 91\%) were observed until occurrence of death $(\mathrm{n}=130)$ or intervention (emergent, $\mathrm{n}=26$; curative, $\mathrm{n}=47$; preemptive, $\mathrm{n}=8$ ). Twenty-two patients, however, were alive and intervention free at last encounter after a median follow-up of 22 months (range, 5 to 48 months) from initiation of chemotherapy, which is nearly double the median time observed for primary tumor complications to manifest. Although it is unlikely that all of these 22 patients would ever require an emergent intervention, we did perform an analysis by excluding them from the nonintervention group, and results were similar. The risk of emergent intervention was not associated with age, primary tumor anatomic location, number of metastatic sites, use of bevacizumab, carcinoembryonic antigen, albumin, lactate dehydrogenase, or alkaline phosphatase levels (Table 2).

Table 1. Time From Initiation of Chemotherapy to Intervention and Survival After Intervention for Patients Who Underwent Interventions and Resections

\begin{tabular}{|c|c|c|c|c|c|c|}
\hline \multirow[b]{2}{*}{ Intervention or Resection } & \multicolumn{4}{|c|}{ Time From Initiation of Chemotherapy to Intervention (months) } & \multicolumn{2}{|c|}{ Survival After Intervention (months)" } \\
\hline & No. & $\%+$ & Median & Range & Median & $95 \% \mathrm{Cl}$ \\
\hline Operative intervention & 16 & 7 & 7 & $1-27$ & 6 & 4 to 27 \\
\hline Nonoperative intervention & 10 & 4 & 12 & $1-36$ & 8 & 4 to NA \\
\hline Curative resection & 47 & 20 & 8 & $5-32$ & 44 & 25 to NA $\neq$ \\
\hline Preemptive resection & 8 & 3 & 9 & $5-24$ & 15 & 14 to 24 \\
\hline
\end{tabular}

NOTE. Median survival from initiation of chemotherapy for the 152 patients who never required an intervention was 13 months (95\% $\mathrm{Cl}$, 10 to 16 months).

Abbreviation: NA, not available.

*Kaplan-Meier estimates of median survival.

tReported as a percentage of the entire cohort.

fUpper limit not calculable. 


\begin{tabular}{|c|c|c|c|c|c|}
\hline \multirow[b]{2}{*}{ Variable } & \multicolumn{2}{|c|}{$\begin{array}{c}\text { No } \\
(n=207)^{*}+ \\
\end{array}$} & \multicolumn{2}{|c|}{$\begin{array}{c}\text { Yes } \\
(\mathrm{n}=26)\end{array}$} & \multirow[b]{2}{*}{$P$} \\
\hline & No. & $\%$ & No. & $\%$ & \\
\hline Age $>60$ years & 108 & 52 & 9 & 35 & .10 \\
\hline Tumor in rectum ( $v$ colon) & 66 & 32 & 12 & 46 & .19 \\
\hline Bevacizumab treatment & 100 & 48 & 12 & 46 & $>.99$ \\
\hline$\geq$ Two sites of metastasis & 120 & 58 & 19 & 73 & .20 \\
\hline CEA > $200 \mathrm{ng} / \mathrm{mL}$ & 95 & 47 & 8 & 31 & .18 \\
\hline Albumin $<3 \mathrm{~g} / \mathrm{dL}$ & 29 & 15 & 4 & 15 & $>.99$ \\
\hline LDH > $200 \mathrm{IU} / \mathrm{L}$ & 72 & 65 & 12 & $60 \neq$ & .80 \\
\hline $\begin{array}{l}\text { Alkaline phosphatase } \\
\quad>250 \mathrm{IU} / \mathrm{L}\end{array}$ & 51 & 25 & 4 & 15 & .33 \\
\hline \multicolumn{6}{|c|}{$\begin{array}{l}\text { Abbreviations: CEA, carcinoembryonic antigen; LDH, lactate dehydrogenase. } \\
\text { *This group includes } 22 \text { patients who were alive and intervention free at last } \\
\text { encounter. These } 22 \text { patients had a median follow-up time of } 22 \text { months from } \\
\text { initiation of chemotherapy, which is almost double the median time observed } \\
\text { for emergent intervention to become necessary. After these } 22 \text { patients were } \\
\text { excluded from the analysis, results were similar. } \\
\text { †Total No. of patients in the no-intervention group differed for the following } \\
\text { variables: CEA }(n=203 \text { ), albumin ( } n=198), L D H(n=113 \text { ), and alkaline } \\
\text { phosphatase }(n=205) \text {. } \\
\neq \text { Total No. of patients }=20 \text {. }\end{array}$} \\
\hline
\end{tabular}

\section{DISCUSSION}

The main finding of this study was that, among patients with metastatic colorectal cancer and a confirmed intact primary tumor who received up-front, modern combination chemotherapy, the incidence of major complications that involved the primary tumor and that required surgery was low. Of 233 such patients, 93\% never required surgical intervention for primary tumor symptomatology. Seven percent underwent emergent surgery at a median of 7 months from initiation of chemotherapy. An additional $4 \%$ required nonoperative intervention (ie, stent or radiotherapy) at a median of 12 months from initiation of chemotherapy.

These findings are particularly well timed, as two recent large epidemiologic surveys that used the Surveillance Epidemiology and End Results database showed that, in the 1990s, more than two thirds of patients who presented with synchronous, stage IV colorectal cancer in the United States underwent up-front surgical resection of their primary tumors. ${ }^{12,13}$ This practice pattern appears to be based on the quantifiable incidence of late, primary tumor-related events observed in patients with synchronous, stage IV colorectal cancer primarily treated with fluoropyrimidine-only conventional chemotherapy. Specifically, retrospective data from the past decade indicate that these patients were exposed to a $9 \%$ to $29 \%$ risk of requiring subsequent urgent surgical palliation for primary tumor-related complications. ${ }^{14-17}$ Additionally, in a single small, prospective study that addresses this question, six (25\%) of 24 patients treated with up-front fluorouracil and leucovorin chemotherapy required subsequent surgical intervention for primary tumor palliation. ${ }^{18}$ In the 255 patients collectively studied in these five reports, the rate of late surgical intervention necessary to palliate primary tumor-related events was $20 \%$. This number is considerably higher than the $7 \%$ surgical intervention rate observed in our study with the use of modern, oxaliplatin- or irinotecan-based, triple-drug chemotherapy.
Prophylactic surgery has historically been advocated for these patients to prevent acute bowel obstruction or perforation, as emergent surgery is associated with higher mortality. Of the 16 patients in this study who required late emergent surgical palliation, two died in the postoperative period, which represented $0.8 \%$ of the total study population. This number compares favorably with the mortality associated with prophylactic colon resection in the metastatic setting, which ranges from $4.6 \%$ to $10 \%$ in representative series. ${ }^{12,17,19,20}$ Thus, given the limitations of cross-study comparisons, it would certainly appear that the routine use of prophylactic colon resection in this setting merits reevaluation.

Prophylactic primary tumor resection has previously been recommended selectively for patients with low metastatic burden who are presumed to be at high risk of obstruction because of their relatively long survival times. ${ }^{19}$ Other reports, however, have failed to establish a correlation between advanced disease stage and the incidence of late primary tumor complications. ${ }^{17,18}$ This study examined several markers representative of tumor burden (ie, two or more sites of metastasis, carcinoembryonic antigen, alkaline phosphatase, lactate dehydrogenase, albumin) and found no statistically significant association with late primary tumor complications. In addition, neither age, use of the monoclonal antibody bevacizumab, or primary tumor location appeared to influence the rate of emergent intervention (Table 2).

Bevacizumab has been associated with a $1 \%$ to $2 \%$ incidence of gastrointestinal perforation in prospective clinical trials. ${ }^{3,4}$ Virtually all bevacizumab-related perforations were observed in the first 3 months of treatment, and most were within the first month. They occurred throughout the entire gastrointestinal tract, and they hardly ever involved the site of the primary tumor. In this study, approximately half of the patients received bevacizumab and only two of the five perforations observed (all at the site of the primary tumor) occurred during bevacizumab therapy; one patient experienced perforation 6 months after the final administration of bevacizumab, whereas two were naive to this agent. Although the small number of patients who developed this complication precludes definitive conclusions, in our experience, bevacizumab did not appear to increase the rate of perforation.

Similarly, location of the primary tumor in the rectum, as opposed to the colon, was not associated with a higher incidence of late, emergent intervention. Specifically, primary tumors located in the rectum required similar rates of operative $(6 \% v 7 \% ; P=.94)$ and overall ( $15 \% v 9 \%$; $P=.19)$ emergent intervention (Table 2$)$ compared with tumors located in the colon. This finding is particularly relevant. The management of synchronous metastatic rectal cancer in the United States during the previous decade has traditionally included prophylactic use of surgery or radiotherapy in up to $45 \%$ to $62 \%$ and $12 \%$ of patients, respectively, ${ }^{12,13}$ mainly because of concern that the unresected primary tumor could lead to significant pelvic symptoms in the future. Our experience of 78 patients with synchronous, stage IV rectal cancer-none of whom were stented, radiated, or operated on initially, but all of whom received up-front modern, triple-drug combination chemotherapy-suggests that only $6 \%$ required surgery and an additional 9\% required nonoperative intervention (ie, stent or radiotherapy) to palliate primary tumor symptoms. The low incidence of late, symptom-directed intervention does not justify routine use of prophylactic surgery or radiotherapy in this setting. 
We were interested in examining whether this initial nonoperative strategy was associated with a detrimental effect on survival when intervention was required. As summarized in Table 1, the median times to operative and nonoperative interventions were 7 and 12 months, respectively, and the median survival rates after interventions were 6 and 7 months, respectively. Median survival from initiation of chemotherapy for patients who did not require intervention was 13 months. A direct comparison of survival after initiation of chemotherapy between patients who did and did not undergo late intervention is not statistically valid, as intervention is a metachronous event and is not established at time zero. However, these numbers taken together suggest that the need for late intervention-despite being a potential marker of aggressive tumor biology and/or chemoresistance-does not appear associated with worse survival when this is measured from initiation of chemotherapy.

We studied only patients treated at our institution to assure that all complications would be captured. We elected to use intervention rate as the study end point, as all patients were initially evaluated and subsequently observed by both medical and surgical oncology to detect primary-related complications in a timely manner. Every patient who presented with acute symptoms or signs related to a primary tumor underwent treatment by either surgical or nonsurgical intervention (ie, stent or radiotherapy). No patient in this cohort died as a result of acute symptoms of colonic obstruction, perforation, sepsis, or hemorrhage without intervention.

On the basis of the paucity of prospective data on this particular controversy in oncology, ${ }^{21,22}$ the National Surgical Adjuvant Breast and Bowel Project has formulated Protocol C-10, entitled "A phase II Trial of 5-Fluorouracil, Leucovorin, and Oxaliplatin (mFOLFOX6) Chemotherapy Plus Bevacizumab for Patients with Unresectable stage IV Colon Cancer and Synchronous Asymptomatic Primary Tumor." ${ }^{23}$ The trial opened in March 2006 and has an accrual goal of 90 patients with colon cancer. The primary end point is the rate of primary tumor-related events (ie, obstruction, perforation, fistula, hemorrhage) that necessitate surgery. The secondary aim is to determine the rate of specific events related to the intact primary tumor that require hospitalization or major intervention.

In conclusion, of 233 patients with confirmed intact primary tumors who received modern, triple-drug combination chemotherapy for synchronous, stage IV colorectal cancer at MSKCC, 93\% never required surgery to palliate primary tumor-related complications. The finding supports our institutional policy of nonoperative initial management of the asymptomatic primary tumor in patients with synchronous stage IV colorectal cancer. This policy allows prompt initiation of systemic therapy, is associated with a low incidence of late, symptom-directed interventions, and should be regarded as routine standard practice for treating patients with synchronous metastatic colorectal cancer without overt obstruction or hemorrhage.

\section{AUTHORS' DISCLOSURES OF POTENTIAL CONFLICTS OF INTEREST}

Although all authors completed the disclosure declaration, the following author(s) indicated a financial or other interest that is relevant to the subject matter under consideration in this article. Certain relationships marked with a " $U$ " are those for which no compensation was received; those relationships marked with a "C" were compensated. For a detailed description of the disclosure categories, or for more information about ASCO's conflict of interest policy, please refer to the Author Disclosure Declaration and the Disclosures of Potential Conflicts of Interest section in Information for Contributors.

Employment or Leadership Position: None Consultant or Advisory Role: Leonard B. Saltz, Roche (C), Genentech (C), Pfizer (C), Bristol-Myers Squibb (C), ImClone Systems (C), Amgen (C) Stock Ownership: None Honoraria: Nancy E. Kemeny, sanofi-aventis Research Funding: Leonard B. Saltz, Roche, Genentech, Pfizer, Bristol-Myers Squibb, ImClone Systems, Amgen; Nancy E. Kemeny, sanofi-aventis, Pfizer Expert

Testimony: None Other Remuneration: None

\section{AUTHOR CONTRIBUTIONS}

Conception and design: George A. Poultsides, Leonard B. Saltz, Nancy E. Kemeny, W. Douglas Wong, Phillip B. Paty

Administrative support: Leonard B. Saltz, Nancy E. Kemeny,

W. Douglas Wong

Provision of study materials or patients: Leonard B. Saltz, Nancy E. Kemeny, Jose G. Guillem, Martin Weiser, Larissa K.F. Temple, W. Douglas Wong, Phillip B. Paty

Collection and assembly of data: George A. Poultsides, Elliot L. Servais, Nancy E. Kemeny, Phillip B. Paty

Data analysis and interpretation: George A. Poultsides, Elliot L. Servais, Leonard B. Saltz, Sujata Patil, Nancy E. Kemeny, Jose G. Guillem, Martin Weiser, Larissa K.F. Temple, Phillip B. Paty

Manuscript writing: George A. Poultsides, Leonard B. Saltz, Sujata Patil, Nancy E. Kemeny, Larissa K.F. Temple, Phillip B. Paty

Final approval of manuscript: George A. Poultsides, Elliot L. Servais, Leonard B. Saltz, Sujata Patil, Nancy E. Kemeny, Jose G. Guillem, Martin Weiser, Larissa K.F. Temple, W. Douglas Wong, Phillip B. Paty

\section{REFERENCES}

1. Tournigand $C$, Andre $T$, Achille $E$, et al: FOLFIRI followed by FOLFOX6 or the reverse sequence in advanced colorectal cancer: A randomized GERCOR study. J Clin Oncol 22:229-237, 2004

2. Goldberg RM, Sargent DJ, Morton RF, et al: A randomized controlled trial of fluorouracil plus leucovorin, irinotecan, and oxaliplatin combinations in patients with previously untreated metastatic colorectal cancer. J Clin Oncol 22:23-30, 2004

3. Hurwitz $H$, Fehrenbacher L, Novotny W, et al: Bevacizumab plus irinotecan, fluorouracil, and leucovorin for metastatic colorectal cancer. N Engl J Med 350:2335-2342, 2004
4. Giantonio BJ, Catalano PJ, Meropol NJ, et al: Bevacizumab in combination with oxaliplatin, fluorouracil, and leucovorin (FOLFOX4) for previously treated metastatic colorectal cancer: Results from the Eastern Cooperative Oncology Group Study E3200. J Clin Oncol 25:1539-1544, 2007

5. Kabbinavar FF, Hambleton J, Mass RD, et al: Combined analysis of efficacy: The addition of bevacizumab to fluorouracil/leucovorin improves survival for patients with metastatic colorectal cancer. $\mathrm{J}$ Clin Oncol 23:3706-3712, 2005

6. Saltz LB, Cox JV, Blanke C, et al: Irinotecan plus fluorouracil and leucovorin for metastatic colorectal cancer: Irinotecan Study Group. N Engl J Med 343:905-914, 2000

7. de Gramont A, Figer $A$, Seymour $M$, et al: Leucovorin and fluorouracil with or without oxalipla- tin as first-line treatment in advanced colorectal cancer. J Clin Oncol 18:2938-2947, 2000

8. Maindrault-Goebel $F$, Louvet $C$, Andre T, et al: Oxaliplatin added to the simplified bimonthly leucovorin and 5-fluorouracil regimen as second-line therapy for metastatic colorectal cancer (FOLFOX6): GERCOR. Eur J Cancer 35:1338-1342, 1999

9. Douillard JY, Cunningham D, Roth $A D$, et al: Irinotecan combined with fluorouracil compared with fluorouracil alone as first-line treatment for metastatic colorectal cancer: A multicentre randomised trial. Lancet 355:1041-1047, 2000

10. The MSKCC Intranet: Information systems: Institutional database, 6/08 update. http://mskweb5 .mskcc.org/intranet/html/12841.cfm

11. D'Angelica M: Are patients with multiple hepatic metastases from colorectal cancer candidates 
for surgery? Nat Clin Pract Oncol 4:682-683, 2007

12. Temple LK, Hsieh L, Wong WD, et al: Use of surgery among elderly patients with stage IV colorectal cancer. J Clin Oncol 22:3475-3484, 2004

13. Cook AD, Single R, McCahill LE: Surgical resection of primary tumors in patients who present with stage IV colorectal cancer: An analysis of Surveillance, Epidemiology, and End Results data, 1988 to 2000. Ann Surg Oncol 12:637-645, 2005

14. Tebbutt NC, Norman AR, Cunningham D, et al: Intestinal complications after chemotherapy for patients with unresected primary colorectal cancer and synchronous metastases. Gut 52:568573, 2003

15. Ruo L, Gougoutas C, Paty PB, et al: Elective bowel resection for incurable stage IV colorecta cancer: Prognostic variables for asymptomatic patients. J Am Coll Surg 196:722-728, 2003

16. Michel P, Roque I, Di Fiore F, et al: Colorectal cancer with non-resectable synchronous metastases: Should the primary tumor be resected? Gastroenterol Clin Biol 28:434-437, 2004

17. Scoggins $C R$, Meszoely IM, Blanke $C D$, et al: Nonoperative management of primary colorectal cancer in patients with stage IV disease. Ann Surg Oncol 6:651-657, 1999

18. Sarela Al, Guthrie JA, Seymour MT, et al: Non-operative management of the primary tumour in patients with incurable stage IV colorectal cancer. Br J Surg 88:1352-1356, 2001

19. Rosen SA, Buell JF, Yoshida A, et al: Initial presentation with stage IV colorectal cancer: How aggressive should we be? Arch Surg 135:530-534, 2000; discussion 534-535
20. Mella $J$, Biffin $A$, Radcliffe $A G$, et al: Population-based audit of colorectal cancer management in two UK health regions: Colorectal Cancer Working Group, Royal College of Surgeons of England Clinical Epidemiology and Audit Unit. Br J Surg 84:1731-1736, 1997

21. Kemeny MM: Surgery should be the primary treatment of synchronous colorectal metastases in the asymptomatic patient [corrected]. Ann Surg Oncol 13:140-141, 2006

22. Petrelli NJ: Systemic chemotherapy should be the primary treatment of synchronous colorectal metastases in the asymptomatic patient [corrected] Ann Surg Oncol 13:137-139, 2006

23. National Surgical Adjuvant Breast and Bowe Project: Federally supported clinical trials: Protocol C-10, 6/08 update. http://www.nsabp.pitt.edu/C10_ Information.asp 